\title{
Predictors of thrombolysis in the telestroke and non telestroke settings for hypertensive acute ischemic stroke patients
}

\author{
Leanne Brecthel, Jordan Gainey, Alexandria Penwell and Thomas I. Nathaniel ${ }^{*}$ (D)
}

\begin{abstract}
Background: In acute ischemic stroke patients, telestroke technology provides sustainable approaches to improve the use of thrombolysis therapy. How this is achieved as it relates to inclusion or exclusion of clinical risk factors for thrombolysis is not fully understood. We investigated this in a population of hypertensive stroke patients.

Methods: Structured data from a regional stroke registry that contained telestroke and non telestroke patients with a primary diagnosis of acute ischemic stroke with history of hypertension were collected between January 2014 and June 2016. Clinical risk factors associated with inclusion or exclusion for recombinant tissue plasminogen activator (rtPA) in the telestroke and non telestroke were identified using multiple regression analysis. Associations between variables and rtPA in the regression models were determined using variance inflation factors while the fitness of each model was determined using the ROC curve to predict the power of each logistic regression model.

Results: The non telestroke admitted more patients (62\% vs 38\%), when compared with the telestroke. Although the telestroke admitted fewer patients, it excluded $11 \%$ and administered thrombolysis therapy to $89 \%$ of stroke patients with hypertension. In the non telestroke group, adjusted odd ratios showed significant associations of $\mathrm{NIH}$ stroke scale score $(\mathrm{OR}=1.059,95 \% \mathrm{Cl}, 1.025-1.093, P<0.001)$ and coronary artery disease $(\mathrm{OR}=2.003,95 \% \mathrm{Cl}, 1.16-3.457$, $P=0.013)$ with inclusion, while increasing age $(\mathrm{OR}=0.979,95 \% \mathrm{Cl}, 0.961-0.996, P=0.017)$, higher INR $(\mathrm{OR}=0.146$, $95 \% \mathrm{Cl}, 0.032-0.665, P=0.013)$, history of previous stroke ( $\mathrm{OR}=0.39,95 \% \mathrm{Cl}, 0.223-0.68, P=0.001)$, and renal insufficiency $(\mathrm{OR}=0.153,95 \% \mathrm{Cl}, 0.046-0.508, P=0.002)$ were associated with rtPA exclusion. In the telestroke, only direct admission to the telestroke was associated with rtPA administration, $(\mathrm{OR}=4.083,95 \% \mathrm{Cl}, 1.322-12.611, P=0.014)$.

Conclusion: The direct admission of hypertensive stroke patients to the telestroke network was the only factor associated with inclusion for thrombolysis therapy even after adjustment for baseline variables. The telestroke technology provides less restrictive criteria for clinical risk factors associated with the inclusion of hypertensive stroke patients for thrombolysis.
\end{abstract}

Keywords: Acute ischemic stroke, Hypertension, rtPA, Inclusion criteria, Exclusion criteria

\section{Background}

One major attempt to address the rural-to-urban disparity and expand the availability of best stroke practices is the development of telestroke networks for acute stroke evaluation, management and clinical care [1-3]. Telestroke technology works to leverage the expertise of the stroke centers to extend the benefits of systemic thrombolysis to rural areas, and to eliminate inequities

\footnotetext{
* Correspondence: nathanit@greenvillemed.sc.ed

University of South Carolina, School of Medicine-Greenville, Greenville, SC 29605, USA
}

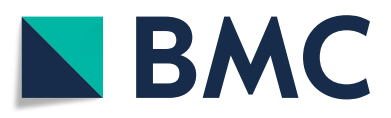

(c) The Author(s). 2018 Open Access This article is distributed under the terms of the Creative Commons Attribution 4.0 International License (http://creativecommons.org/licenses/by/4.0/), which permits unrestricted use, distribution, and reproduction in any medium, provided you give appropriate credit to the original author(s) and the source, provide a link to the Creative Commons license, and indicate if changes were made. The Creative Commons Public Domain Dedication waiver (http://creativecommons.org/publicdomain/zero/1.0/) applies to the data made available in this article, unless otherwise stated. between rural and urban care of stroke patients. The factor of ten over previous numbers when telestroke technology is applied $[4,5]$. This is because telestroke extends the expertise of stroke centers to provide enhanced stroke care, particularly the administration of rtPA to smaller rural and community hospitals [6, 7]. Like all medical novelties, it is faster, employs the most recent technology and promotes a better use of limited resources. 
Moreover, telestroke technology represents a major growth in many hospitals' efforts to reduce door-to-needle time $[3,8]$ outside the traditional role of the telestroke in providing the hub-and-spoke system model for stroke care. Such a centralized role of the telestroke in reducing door-to-needle time may affect treatment times and use of thrombolysis [9-18]. Whether such a centralized role of the telestroke contributes to the reduction in pretreatment clinical risk factors associated with thrombolysis therapy is not very clear. Irrespective of whether the stroke patient is treated in the telestroke or non telestroke, hypertension is the most influential adjustable variable and the second most powerful clinical risk factor after age for stroke [15, 19-21]. Hypertensive patients are 60-70\% more likely to suffer a stroke when compared to those without hypertension [22-25]. The strong relationship between stroke and hypertension has been investigated extensively [18, 23, 26-29]. Findings from these studies result in suggestions for the optimization of various hypertensive medications in hypertensive stroke patients prior to treatment with thrombolysis therapy. High-quality telestroke care remains efficient for all stroke patients [30], and the role of the telestroke is expected to increase with benefits of thrombolysis [31] for stroke patients including those with incidence of hypertension. Past studies have shown that decreases in mean arterial pressure (MAP) have a negative association with cerebral blood flow and patients with chronic hypertension have shifts in perfusion autoregulation parameters as well as changes in collateral blood supplies [32, 33]. This, in conjunction with other considerations such as carotid artery stenosis [34-36], put hypertensive stroke patients at a unique risk special consideration should be considered when determining rtPA eligibility in these patients.

In general, the association of the telestroke technology and thrombolysis therapy with exclusion or inclusion clinical risk factors in hypertensive stroke is not fully understood. Since stroke with hypertension is dynamic and complicated, one possibility is that the telestroke technology may be less or more stringent in the inclusion or exclusion of clinical risk factors for thrombolysis therapy when compared with the non telestroke setting for hypertensive stroke patients. We hypothesize that the practice-based model of telestroke can manage pretreatment clinical risk factors in hypertensive stroke patients for thrombolysis therapy even in the quest for faster growth and, in so doing telestroke may function as a model to relax the criteria for the inclusion or exclusion for thrombolysis in hypertensive stroke patients.

\section{Data collection}

We used structured data collected from electronic health records of patients presenting acute ischemic stroke with history of hypertension between January 2014 and June 2016 from the Greenville Health System Stroke Registry. The stroke registry contained data for telestroke and non telestroke patients with a primary diagnosis of ischemic stroke and has been described in our previous studies [13, 18, 31, 37-40]. All data for the patient's demographics and clinical variables from both the telestroke and non telestroke patients were abstracted by a stroke nurse. Baseline clinical risk factors were retrieved from documented patients' medical history. Data were collected for demographics (age and gender), admission date, medications, clinical diagnosis, prehospital care, pre-stroke and post-stroke ambulatory status, in-hospital procedures, past medical history. All data were scrutinized under quality control checks using established protocol to determine the quality of the data and to insure against several types of errors, including: errors in interpretation or coding, errors in data entry. All data including neuroimaging data were reviewed by a clinician, who determined whether the patient met the clinical case description of acute stroke. The events were categorized as ischemic stroke, transient ischemic attack, intracerebral hemorrhage, or subarachnoid hemorrhage according to descriptions of stroke from the Classification of Cerebrovascular Diseases III [41]. Patients with missing brain imaging data or no information for times of receiving $\mathrm{rtPA}$, and stroke patients with incidence hypertension without medical records were all excluded. In addition, we excluded data from patients that received endovascular therapy to maintain homogeneity of the data. Patients with a history of hypertension have been described in our previous study [23]. This is based on the guideline for the management of hypertension pre and post stroke according to Joint National Committee (JNC7) guideline, stroke with hypertension (stages 1;SBP; 140-159, DBP 90-99 and stage 2;SBP,> 160, DBP; or $>100$ ) should be managed to levels or $130 / 80 \mathrm{mmHg}$ or lower [42].

In addition, we collected data on symptom onset time and the admission to Emergency Department (ED) for both telestroke and non telestroke hypertensive stroke patients. We identified patients directly admitted to the ED or with emergency medical services (EMS). Patients with indirect admission by being transferred to the ED in the telestroke or non telestroke from another hospital were also identified. Onset time referred to the time that the patient first presented with neurological disorder or the last normal observation for unknown clinical conditions. We collected baseline information for NIHSS score, pre-rtPA systolic and diastolic blood pressure, and the presence of comorbid risk factors, including a history of diabetes mellitus, prior stroke or transient ischemic attack, and atrial fibrillation. Additional medical history including information on carotid artery stenosis, 
hypertension, prosthetic heart valve, renal insufficiency, smoking, sleep apnea, migraine, obesity, and peripheral vascular disease was collected. Information of demographics include age, sex, race, and ethnicity. Information of laboratory analysis was collected including total cholesterol, triglycerides, HDL, LDL, lipids, blood glucose, and creatine. Information on ambulation status prior to event, during and at discharge were also collected.

\section{Data analysis}

All statistical analyses were performed utilizing SPSS Statistics Software version 15.0 (Chicago, IL) and $P<$ 0.05 was used to establish statistical significance in all comparisons between groups. A univariate analysis was used to determine factors that were associated with inclusion or exclusion for rtPA in the population of hypertensive stroke patients. Descriptive statistics were calculated for the demographic and clinical characteristics of patients. All continuous variables are represented as mean \pm standard deviation and comparisons between groups were determined using a Student's T-test. All discrete variables are represented as number (percentage) and comparisons between groups were made using Pearson's Chi-Squared analyses. Multivariate analysis was performed to determine demographic and clinical characteristics that were more associated with the telestroke subgroup utilizing a stepwise conditional logistic regression with $p<0.05$. Univariate analysis was repeated to determine factors associated with inclusion for rtPA in the separate telestroke subgroup and non-telestroke subgroup for hypertensive stroke patients. Multivariate analysis using a stepwise conditional logistic regression with $p<0.05$ was performed to determine demographic and clinical characteristics associated with exclusion from rtPA administration in the total study population as well as in the telestroke subgroup and non-telestroke subgroup. All stepwise regression models were assessed using Hosmer \& Lemeshow test, Cox \& Snell $\mathrm{R}^{2}$ and Classification Plots and ROC analysis. Multicollinearity of variables were assessed with variance inflation factor analysis to confirm independence of variables included in the regression model. We presented all results for our multivariate analysis as odds ratio estimates at $95 \%$ confidence interval and results were considered significant at $P<0.05$.

\section{Results}

A total of 434 stroke patients with hypertension were admitted to the non telestroke while 267 were admitted in the telestroke. Comparisons between the baseline demographic and clinical characteristics of telestroke and non-telestroke patients are presented in Table 1. Compared to the non-telestroke control group, telestroke patients were younger $(66.1 \pm 13.5$ vs. $70.1 \pm 14.1)$, included more
African-American or other minority group individuals (19.1\% vs. $12.2 \%)$, and have a higher body mass index (30.8 \pm 7.3 vs. $28.2 \pm 6.7$ ). Prior to admission, telestroke patients had lower rates of atrial fibrillation or atrial flutter (11.6\% vs. 27.2\%) and were less likely to have had a previous stroke ( $24.7 \%$ vs. $32.0 \%)$, but more likely to have diabetes $(46.1 \%$ vs. $38.0 \%$ ), depression ( $18.0 \%$ vs $0.2 \%)$, hormone replacement therapy ( $3 \% 0$ vs $0.7 \%$ ) and more likely to be currently medicated for diabetes (37.1\% vs. $27.4 \%)$. Initial labs at the time of presentation showed that telestroke patients tended to have lower creatinine $(1.2 \pm 0.8$ vs. $1.4 \pm 1.1)$, lower INR (1.0 \pm 0.2 vs. $1.1 \pm 0.5)$, lower heart rate $(78.2 \pm 15.9$ vs. $82.7 \pm$ $18.5)$, systolic blood pressure $(148.3 \pm 24$ vs $155.9 \pm 30.9)$, and diastolic blood pressure $(79.8 \pm 16.3$ vs. $83.6 \pm 20.6)$. Telestroke patients also had a better ambulatory status prior to admission, at the time of presentation, and at discharge, were more likely to receive rtPA ( $89.1 \%$ vs. $38.7 \%)$ and were more likely to experience an improved ambulatory status at discharge relative to presentation ( $70.4 \%$ vs. $58.1 \%)$.

Clinical characteristics associated with the inclusion and exclusion for rtPA for patients with a history of hypertension in the telestroke and non-telestroke cohorts are presented in Table 2. Of the 434 that were admitted in the non telestroke $61.3 \%$ were excluded from rtPA while $38.7 \%$ received rtPA. For the telestroke, 10.9\% were excluded while $89.1 \%$ hypertensive stroke patients received rtPA. In the non-telestroke, patients who received rtPA were younger than patients who did not receive rtPA $(68 \pm 14.3$ vs. $71.4 \pm 13.9$ ). Patients who received rtPA outside of a telestroke network presented with lower rates of carotid artery stenosis $(2.4 \%$ vs. $7.9 \%)$, congestive heart failure $(9.5 \%$ vs. $16.9 \%)$, previous stroke ( $23.2 \%$ vs. $37.6 \%)$, prosthetic heart valve ( $0 \%$ vs. $3.4 \%)$, renal insufficiency ( $3.0 \%$ vs. $10.5 \%)$, and lower blood glucose level $(138.6 \pm 68.7$ vs. $158.9 \pm 95.1)$. Patients treated with rtPA outside of the telestroke network also presented with a lower creatinine $(1.2 \pm 0.5$ vs. $1.5 \pm$ $1.4)$, and INR ( $1.1 \pm 0.1$ vs. $1.2 \pm 0.6)$. Patients who received rtPA were more likely to experience improvement in their ambulatory status from presentation to discharge (65.5\% vs. $53.4 \%)$. Within the telestroke subgroup there were fewer statistically significant differences between patients who received rtPA and patients who were excluded from rtPA. Patients who received rtPA were more likely to have a family history of stroke ( $13.4 \%$ vs $0 \%)$.

Following the use of multivariate analysis to adjust for the cofounding effects of comorbidities in the telestroke and non-telestroke (Table 3), patients that present with obesity (OR $=2.351,95 \%$ CI, 1.352-4.087, $P=0.002)$ were directly admitted for treatment ( $\mathrm{OR}=$ 32.855, 95\% CI, 15.706-68.727, $P<0.001)$ and received rtPA $\quad(\mathrm{OR}=5.199, \quad 95 \%$ CI $, 2.647-10.211, \quad P<0.001)$ have higher odds of being associated with the telestroke, while higher systolic blood pressure $(\mathrm{OR}=$ 0.987, 95\% CI, 0.977-0.997, $P=0.01$ ) was associated 
Table 1 Demographic factors and clinical characteristics of acute ischemic stroke patients with a history of hypertension divided by telestroke status

\begin{tabular}{|c|c|c|c|}
\hline Characteristic & Non-Telestroke $(N=434)$ & Telestroke $(N=267)$ & $P$-Value \\
\hline \multicolumn{4}{|l|}{ Patient Age in Years } \\
\hline Mean \pm SD & $70.1 \pm 14.1$ & $66.1 \pm 13.5$ & $<0.001^{*}$ \\
\hline \multicolumn{4}{|l|}{ Age Group: No. (\%) } \\
\hline$<50$ years & $45(10.4)$ & $31(11.6)$ & \multirow[t]{5}{*}{$0.002^{*}$} \\
\hline $50-59$ & $56(12.9)$ & $47(17.6)$ & \\
\hline $60-69$ & $88(20.3)$ & $76(28.5)$ & \\
\hline $70-79$ & $118(27.2)$ & $67(25.1)$ & \\
\hline$\geq 80$ & $127(29.3)$ & $46(17.2)$ & \\
\hline \multicolumn{4}{|l|}{ Gender: No. (\%) } \\
\hline Male & $203(46.8)$ & $139(52.1)$ & \multirow[t]{2}{*}{0.174} \\
\hline Female & $231(53.2)$ & $128(47.9)$ & \\
\hline \multicolumn{4}{|l|}{ Race: No. (\%) } \\
\hline Caucasian & $346(79.7)$ & $209(78.3)$ & \multirow[t]{3}{*}{$0.010^{*}$} \\
\hline African-American & $50(11.5)$ & $46(17.2)$ & \\
\hline Other & $3(0.7)$ & $5(1.9)$ & \\
\hline Hispanic Ethnicity: No. (\%) & $5(1.2)$ & $6(2.2)$ & 0.257 \\
\hline \multicolumn{4}{|l|}{ Body Mass Index } \\
\hline Mean \pm SD & $28.2 \pm 6.7$ & $30.8 \pm 7.3$ & $<0.001^{*}$ \\
\hline \multicolumn{4}{|l|}{ Medical History: No. (\%) } \\
\hline Atrial Fib/Flutter & $118(27.2)$ & $31(11.6)$ & $<0.001^{*}$ \\
\hline Carotid Artery Stenosis & $25(5.8)$ & $16(6)$ & 0.899 \\
\hline Congestive Heart Failure & $61(14.1)$ & $33(12.4)$ & 0.522 \\
\hline Coronary Artery Disease & $173(39.9)$ & $106(39.7)$ & 0.966 \\
\hline Depression & $1(0.2)$ & $48(18)$ & $<0.001^{*}$ \\
\hline Diabetes & $165(38)$ & $123(46.1)$ & $0.035^{*}$ \\
\hline Dyslipidemia & $259(59.7)$ & $168(62.9)$ & 0.393 \\
\hline Family History of Stroke & $35(8.1)$ & $32(12)$ & 0.086 \\
\hline Hormone Replacement Therapy & $3(0.7)$ & $8(3)$ & $0.017^{*}$ \\
\hline Migraine & $7(1.6)$ & $8(3)$ & 0.219 \\
\hline Obesity & $36(8.3)$ & $25(9.4)$ & 0.626 \\
\hline Peripheral Vascular Disease & (0) & (0) & \\
\hline Previous Stroke & $139(32)$ & $66(24.7)$ & $0.039 *$ \\
\hline Previous TIA & 59 (13.6) & $32(12)$ & 0.538 \\
\hline Prosthetic Heart Valve & $9(2.1)$ & $1(0.4)$ & 0.065 \\
\hline Renal Insufficiency & $33(7.6)$ & $17(6.4)$ & 0.537 \\
\hline Sleep Apnea & $0(0)$ & $12(4.5)$ & $<0.001^{*}$ \\
\hline Smoking & $115(26.5)$ & $67(25.1)$ & 0.681 \\
\hline Substance Abuse & $17(3.9)$ & $7(2.6)$ & 0.36 \\
\hline \multicolumn{4}{|l|}{ Initial NIH Stroke Scale } \\
\hline Mean $\pm S D$ & $10.7 \pm 8.5$ & $9.5 \pm 8.2$ & 0.076 \\
\hline \multicolumn{4}{|l|}{ Initial Labs \& Vitals } \\
\hline Total Cholesterol & $164.9 \pm 48.9$ & $166.6 \pm 42.4$ & 0.651 \\
\hline Triglycerides & $133.2 \pm 89.5$ & $147.7 \pm 103$ & 0.063 \\
\hline $\mathrm{HDL}$ & $40.9 \pm 13.9$ & $40.2 \pm 12.7$ & 0.547 \\
\hline
\end{tabular}


Table 1 Demographic factors and clinical characteristics of acute ischemic stroke patients with a history of hypertension divided by telestroke status (Continued)

\begin{tabular}{|c|c|c|c|}
\hline Characteristic & Non-Telestroke $(N=434)$ & Telestroke $(N=267)$ & $P$-Value \\
\hline LDL & $98.1 \pm 37.1$ & $100.3 \pm 35.3$ & 0.443 \\
\hline Lipids & $6.4 \pm 1.7$ & $6.5 \pm 1.7$ & 0.659 \\
\hline Blood Glucose & $151.1 \pm 86.4$ & $138.9 \pm 72.4$ & 0.058 \\
\hline Creatinine & $1.4 \pm 1.1$ & $1.2 \pm 0.8$ & $0.006^{*}$ \\
\hline INR & $1.1 \pm 0.5$ & $1 \pm 0.2$ & $<0.001^{*}$ \\
\hline Heart Rate & $82.7 \pm 18.5$ & $78.2 \pm 15.9$ & $0.001^{*}$ \\
\hline Systolic Blood Pressure & $155.9 \pm 30.9$ & $148.3 \pm 24$ & $<0.001^{*}$ \\
\hline Diastolic Blood Pressure & $83.6 \pm 20.6$ & $79.8 \pm 16.3$ & $0.007^{*}$ \\
\hline \multicolumn{4}{|c|}{ Medications Prior to Admission: No. (\%) } \\
\hline Antiplatelet or Anticoagulant & $256(59)$ & $151(56.6)$ & 0.526 \\
\hline Antihypertensive & $369(85)$ & $233(87.3)$ & 0.408 \\
\hline Cholesterol Reducer & $222(51.2)$ & $148(55.4)$ & 0.27 \\
\hline Diabetic Medication & $119(27.4)$ & $99(37.1)$ & $0.007^{*}$ \\
\hline \multicolumn{4}{|c|}{ Ambulation Status Prior to Event: No. (\%) } \\
\hline Ambulate Independently & $377(86.9)$ & $252(94.4)$ & \multirow[t]{4}{*}{$0.012^{*}$} \\
\hline Ambulate With Assistance & $22(5.1)$ & $4(1.5)$ & \\
\hline Unable to Ambulate & $19(4.4)$ & $7(2.6)$ & \\
\hline Not Documented & $16(3.7)$ & $4(1.5)$ & \\
\hline \multicolumn{4}{|c|}{ Ambulation Status on Admission: No. (\%) } \\
\hline Ambulate Independently & $60(13.8)$ & $55(20.6)$ & \multirow[t]{4}{*}{$0.005^{*}$} \\
\hline Ambulate With Assistance & $63(14.5)$ & $53(19.9)$ & \\
\hline Unable to Ambulate & $162(37.3)$ & $72(27)$ & \\
\hline Not Documented & $149(34.3)$ & $87(32.6)$ & \\
\hline \multicolumn{4}{|c|}{ Ambulation Status on Discharge: No. (\%) } \\
\hline Ambulate Independently & $162(37.3)$ & $132(49.4)$ & \multirow[t]{4}{*}{$<0.001$} \\
\hline Ambulate With Assistance & $128(29.5)$ & $84(31.5)$ & \\
\hline Unable to Ambulate & $102(23.5)$ & $33(12.4)$ & \\
\hline Not Documented & $42(9.7)$ & $18(6.7)$ & \\
\hline \multicolumn{4}{|l|}{ First Care Received: No. (\%) } \\
\hline Emergency Department & $395(91)$ & $78(29.2)$ & \multirow[t]{2}{*}{$<0.001^{*}$} \\
\hline Direct Admission & $39(9)$ & $189(70.8)$ & \\
\hline rtPA Administration & $168(38.7)$ & $238(89.1)$ & $<0.001^{*}$ \\
\hline Improved Ambulation & $252(58.1)$ & $188(70.4)$ & $0.001^{*}$ \\
\hline
\end{tabular}

Continuous variables are represented as Mean \pm S.D. and comparisons between groups are made with a Student's T Test. Discrete variables are represented as Count (Percent Frequency) and comparisons between groups were made using Pearson's Chi-Squared ${ }^{*} P<0.05$

with the non telestroke. The Receiver Operating Characteristics (ROC) curve for the predictive power of the regression model is presented in Fig. 1. The discriminating capability of the model was very good as shown by the ROC curve, with area under the curve (AUROC) of 0.900 (95\% CI, 0.875-0.924, $P<0.001$ ). Further adjusted analysis was performed focusing on the whole stroke population (Table 4). This analysis determined clinical and demographic factors that were associated with inclusion for rtPA, irrespective of whether patients were treated in the telestroke or non telestroke setting. The results indicate that telestroke as a variable was the strongest predictor of rtPA administration $(\mathrm{OR}=5.204, \quad 95 \%$ CI, $2.582-$ 10.492, $P<0.001$ ), followed by a direct admission $(\mathrm{OR}=4.557,95 \% \mathrm{CI}, 1.772-11.721, P=0.002)$, and higher NIH stroke scale score $(\mathrm{OR}=1.046,95 \% \mathrm{CI}$, 1.016-1.076, $P=0.002)$. A higher INR, $(\mathrm{OR}=0.203$, 
Table 2 Clinical characteristics, medical history, and presenting symptoms of acute ischemic stroke patients with a history of hypertension stratified by rtPA status and telestroke status

\begin{tabular}{|c|c|c|c|c|c|c|}
\hline \multirow[t]{2}{*}{ Characteristic } & \multicolumn{3}{|l|}{ Non-Telestroke } & \multicolumn{3}{|l|}{ Telestroke } \\
\hline & No rtPA $(N=266)$ & $\operatorname{rtPA}(N=168)$ & $P$-Value & No rtPA $(N=29)$ & $\operatorname{rtPA}(N=238)$ & $P$-Value \\
\hline \multicolumn{7}{|l|}{ Patient Age in Years } \\
\hline Mean \pm SD & $71.4 \pm 13.9$ & $68 \pm 14.3$ & $0.015^{*}$ & $65.9 \pm 13.6$ & $66.1 \pm 13.5$ & 0.932 \\
\hline \multicolumn{7}{|l|}{ Age Group: No. (\%) } \\
\hline$<50$ years & $27(10.2)$ & $18(10.7)$ & $0.007^{*}$ & $2(6.9)$ & $29(12.2)$ & 0.268 \\
\hline $50-59$ & $25(9.4)$ & $31(18.5)$ & & $9(31)$ & $38(16)$ & \\
\hline $60-69$ & $47(17.7)$ & $41(24.4)$ & & $7(24.1)$ & $69(29)$ & \\
\hline 70-79 & $83(31.2)$ & $35(20.8)$ & & $5(17.2)$ & $62(26.1)$ & \\
\hline$\geq 80$ & $84(31.6)$ & $43(25.6)$ & & $6(20.7)$ & $40(16.8)$ & \\
\hline \multicolumn{7}{|l|}{ Gender: No. (\%) } \\
\hline Male & $121(45.5)$ & $82(48.8)$ & 0.499 & $15(51.7)$ & $124(52.1)$ & 0.969 \\
\hline Female & $145(54.5)$ & $86(51.2)$ & & $14(48.3)$ & $114(47.9)$ & \\
\hline \multicolumn{7}{|l|}{ Race: No. (\%) } \\
\hline Caucasian & $207(77.8)$ & $139(82.7)$ & 0.451 & $22(75.9)$ & $187(78.6)$ & 0.725 \\
\hline African-American & $33(12.4)$ & $17(10.1)$ & & $4(13.8)$ & $42(17.6)$ & \\
\hline Other & $3(1.1)$ & $0(0)$ & & $1(3.4)$ & $4(1.7)$ & \\
\hline Hispanic Ethnicity: No. (\%) & $3(1.1)$ & $2(1.2)$ & 0.952 & $0(0)$ & $6(2.5)$ & 0.387 \\
\hline \multicolumn{7}{|l|}{ Body Mass Index } \\
\hline Mean \pm SD & $28 \pm 6.7$ & $28.6 \pm 6.7$ & 0.312 & $31.5 \pm 7.2$ & $30.7 \pm 7.3$ & 0.596 \\
\hline \multicolumn{7}{|l|}{ Medical History: No. (\%) } \\
\hline Atrial Fib/Flutter & $81(30.5)$ & $37(22)$ & 0.055 & $4(13.8)$ & $27(11.3)$ & 0.698 \\
\hline Carotid Artery Stenosis & $21(7.9)$ & $4(2.4)$ & $0.016^{*}$ & $1(3.4)$ & $15(6.3)$ & 0.541 \\
\hline Congestive Heart Failure & $45(16.9)$ & $16(9.5)$ & $0.031^{*}$ & $6(20.7)$ & $27(11.3)$ & 0.149 \\
\hline Coronary Artery Disease & $103(38.7)$ & $70(41.7)$ & 0.542 & $11(37.9)$ & $95(39.9)$ & 0.837 \\
\hline Depression & $1(0.4)$ & $0(0)$ & 0.426 & $4(13.8)$ & $44(18.5)$ & 0.534 \\
\hline Diabetes & $103(38.7)$ & $62(36.9)$ & 0.704 & $16(55.2)$ & $107(45)$ & 0.297 \\
\hline Dyslipidemia & $160(60.2)$ & $99(58.9)$ & 0.8 & $16(55.2)$ & $152(63.9)$ & 0.36 \\
\hline Family History of Stroke & $22(8.3)$ & $13(7.7)$ & 0.843 & $0(0)$ & $32(13.4)$ & $0.035^{*}$ \\
\hline Hormone Replacement Therapy & $3(1.1)$ & $0(0)$ & 0.167 & $0(0)$ & $8(3.4)$ & 0.316 \\
\hline Migraine & $2(0.8)$ & $5(3)$ & 0.073 & $1(3.4)$ & $7(2.9)$ & 0.88 \\
\hline Obesity & $93(35)$ & $58(34.5)$ & 0.926 & $15(51.7)$ & $137(57.6)$ & 0.549 \\
\hline Peripheral Vascular Disease & $22(8.3)$ & $14(8.3)$ & 0.982 & $1(3.4)$ & $24(10.1)$ & 0.247 \\
\hline Previous Stroke & $100(37.6)$ & $39(23.2)$ & $0.002^{*}$ & $7(24.1)$ & $59(24.8)$ & 0.939 \\
\hline Previous TIA & $32(12)$ & $27(16.1)$ & 0.231 & $4(13.8)$ & $28(11.8)$ & 0.751 \\
\hline Prosthetic Heart Valve & $9(3.4)$ & $0(0)$ & $0.016^{*}$ & $0(0)$ & $1(0.4)$ & 0.727 \\
\hline Renal Insufficiency & $28(10.5)$ & $5(3)$ & $0.004^{*}$ & $1(3.4)$ & $16(6.7)$ & 0.495 \\
\hline Sleep Apnea & $0(0)$ & $0(0)$ & N/A & $1(3.4)$ & $11(4.6)$ & 0.773 \\
\hline Smoking & $62(23.3)$ & $53(31.5)$ & 0.058 & $9(31)$ & $58(24.4)$ & 0.434 \\
\hline Substance Abuse & $11(4.1)$ & $6(3.6)$ & 0.768 & $1(3.4)$ & $6(2.5)$ & 0.768 \\
\hline \multicolumn{7}{|l|}{ Initial NIH Stroke Scale } \\
\hline Mean \pm SD & $10.1 \pm 9$ & $11.5 \pm 7.9$ & 0.124 & $9.2 \pm 8.3$ & $9.5 \pm 8.3$ & 0.851 \\
\hline
\end{tabular}


Table 2 Clinical characteristics, medical history, and presenting symptoms of acute ischemic stroke patients with a history of hypertension stratified by rtPA status and telestroke status (Continued)

\begin{tabular}{|c|c|c|c|c|c|c|}
\hline \multirow[t]{2}{*}{ Characteristic } & \multicolumn{3}{|l|}{ Non-Telestroke } & \multicolumn{3}{|l|}{ Telestroke } \\
\hline & No rtPA $(N=266)$ & rtPA $(N=168)$ & $P$-Value & No rtPA $(N=29)$ & rtPA $(N=238)$ & $P$-Value \\
\hline \multicolumn{7}{|l|}{ Initial Labs \& Vitals } \\
\hline Total Cholesterol & $165 \pm 52.8$ & $164.7 \pm 43.2$ & 0.956 & $180.7 \pm 50.2$ & $164.9 \pm 41.2$ & 0.068 \\
\hline Triglycerides & $132 \pm 89.5$ & $134.9 \pm 89.8$ & 0.759 & $147.4 \pm 72.9$ & $147.7 \pm 106.1$ & 0.987 \\
\hline $\mathrm{HDL}$ & $40.7 \pm 14.6$ & $41.1 \pm 13$ & 0.834 & $42 \pm 14.1$ & $40 \pm 12.5$ & 0.44 \\
\hline LDL & $97.9 \pm 36.7$ & $98.3 \pm 37.8$ & 0.91 & $111.6 \pm 45.3$ & $99 \pm 33.8$ & 0.08 \\
\hline Lipids & $6.5 \pm 1.8$ & $6.3 \pm 1.5$ & 0.215 & $6.7 \pm 1.9$ & $6.4 \pm 1.7$ & 0.539 \\
\hline Blood Glucose & $158.9 \pm 95.1$ & $138.6 \pm 68.7$ & $0.011^{*}$ & $164.4 \pm 113.6$ & $135.9 \pm 65.4$ & 0.203 \\
\hline Creatinine & $1.5 \pm 1.4$ & $1.2 \pm 0.5$ & $0.001^{*}$ & $1.4 \pm 1.8$ & $1.1 \pm 0.5$ & 0.356 \\
\hline INR & $1.2 \pm 0.6$ & $1.1 \pm 0.1$ & $0.001^{*}$ & $1.1 \pm 0.4$ & $1 \pm 0.1$ & 0.588 \\
\hline Heart Rate & $83.4 \pm 19$ & $81.5 \pm 17.7$ & 0.284 & $76 \pm 13.9$ & $78.4 \pm 16.2$ & 0.444 \\
\hline Systolic Blood Pressure & $154.5 \pm 30.7$ & $158.3 \pm 31.2$ & 0.217 & $152 \pm 24.9$ & $147.8 \pm 23.8$ & 0.38 \\
\hline Diastolic Blood Pressure & $82.6 \pm 21$ & $85.2 \pm 19.8$ & 0.214 & $78.8 \pm 13.8$ & $79.9 \pm 16.6$ & 0.721 \\
\hline \multicolumn{7}{|c|}{ Medications Prior to Admission: No. (\%) } \\
\hline Antiplatelet or Anticoagulant & $162(60.9)$ & $94(56)$ & 0.307 & $18(62.1)$ & $133(55.9)$ & 0.526 \\
\hline Antihypertensive & $222(83.5)$ & $147(87.5)$ & 0.25 & $25(86.2)$ & $208(87.4)$ & 0.856 \\
\hline Cholesterol Reducer & $137(51.5)$ & $85(50.6)$ & 0.854 & $18(62.1)$ & $130(54.6)$ & 0.446 \\
\hline Diabetic Medication & $74(27.8)$ & $45(26.8)$ & 0.814 & $11(37.9)$ & $88(37)$ & 0.92 \\
\hline \multicolumn{7}{|c|}{ Ambulation Status Prior to Event: No. (\%) } \\
\hline Ambulate Independently & $218(82)$ & $159(94.6)$ & $0.002^{*}$ & $25(86.2)$ & $227(95.4)$ & 0.246 \\
\hline Ambulate With Assistance & $18(6.8)$ & $4(2.4)$ & & $1(3.4)$ & $3(1.3)$ & \\
\hline Unable to Ambulate & $16(6)$ & $3(1.8)$ & & $2(6.9)$ & $5(2.1)$ & \\
\hline Not Documented & $14(5.3)$ & $2(1.2)$ & & $1(3.4)$ & $3(1.3)$ & \\
\hline \multicolumn{7}{|c|}{ Ambulation Status on Admission: No. (\%) } \\
\hline Ambulate Independently & $46(17.3)$ & $14(8.3)$ & $<0.001^{*}$ & $11(37.9)$ & $44(18.5)$ & $0.023^{*}$ \\
\hline Ambulate With Assistance & $48(18)$ & $15(8.9)$ & & $4(13.8)$ & 49 (20.6) & \\
\hline Unable to Ambulate & $95(35.7)$ & $67(39.9)$ & & $10(34.5)$ & $62(26.1)$ & \\
\hline Not Documented & $77(28.9)$ & $72(42.9)$ & & $4(13.8)$ & $83(34.9)$ & \\
\hline \multicolumn{7}{|c|}{ Ambulation Status on Discharge: No. (\%) } \\
\hline Ambulate Independently & $94(35.3)$ & $68(40.5)$ & 0.45 & $15(51.7)$ & $117(49.2)$ & 0.736 \\
\hline Ambulate With Assistance & $78(29.3)$ & $50(29.8)$ & & $7(24.1)$ & $77(32.4)$ & \\
\hline Unable to Ambulate & $64(24.1)$ & $38(22.6)$ & & $4(13.8)$ & $29(12.2)$ & \\
\hline Not Documented & $30(11.3)$ & $12(7.1)$ & & $3(10.3)$ & $15(6.3)$ & \\
\hline \multicolumn{7}{|l|}{ First Care Received: No. (\%) } \\
\hline Emergency Department & $242(91)$ & $153(91.1)$ & 0.973 & $18(62.1)$ & $60(25.2)$ & $<0.001^{*}$ \\
\hline Direct Admission & $24(9)$ & $15(8.9)$ & & $11(37.9)$ & $178(74.8)$ & \\
\hline Improved Ambulation & $142(53.4)$ & $110(65.5)$ & $0.013^{*}$ & $20(69)$ & $168(70.6)$ & 0.857 \\
\hline
\end{tabular}

Continuous variables are represented as Mean \pm S.D. and comparisons between groups are made with a Student's T Test. Discrete variables are represented as Count (Percent Frequency) and comparisons between groups were made using Pearson's Chi-Squared ${ }^{*} P<0.05$

95\% CI, $0.06-0.691, P=0.011)$, history of previous stroke $(\mathrm{OR}=0.476,95 \% \mathrm{CI}, 0.293-0.775, P=0.003)$, and renal insufficiency, $(\mathrm{OR}=0.351,95 \% \mathrm{CI}, 0.143-$ $0.858, P=0.022)$, were predictive of exclusion of stroke patients with hypertension from rtPA. As shown in Fig. 2, the predictive power of the logistic regression was strong. The area under the curve (AUROC) is $0.792(95 \% \mathrm{CI}, 0.753-0.830, P<0.05)$. 
Table 3 A stepwise regression model to elucidate clinical factors more associated with acute ischemic stroke patients presenting via telestroke

\begin{tabular}{lllll}
\hline & B Value & Adj. Odds Ratio & Wald & \\
\hline Systolic Blood Pressure & -0.013 & $0.987(0.977-0.997)$ & 6.549 & \\
Obesity & 0.855 & $2.351(1.352-4.087)$ & 9.183 & $0.01^{*}$ \\
Direct Admission & 3.492 & $32.855(15.706-68.727)$ & 86.004 & $0.002^{*}$ \\
rtPA Administration & 1.649 & $5.199(2.647-10.211)$ & 22.916 & $<0.001^{*}$ \\
Constant & -1.003 & 0.367 & 1.401 & $<0.001^{*}$ \\
\hline
\end{tabular}

Positive B values (Adj, OR > 1) denote variables more associated with telestroke patients while negative $B$ values (Adj. OR $<1$ ) denote variables more associated with non-telestroke patients. Multicollinearity and interactions among independent variables were checked. Hosmer-Lemeshow test $(P=0.089)$, Cox \& Snell $\left(R^{2}=0.432\right)$, Classification table (overall correctly classified percentage $=85.3 \%$ ) were applied to check the model fitness ${ }^{*} P<0.05$

In the non-telestroke subgroup (Table 5), higher $\mathrm{NIH}$ stroke scale score $(\mathrm{OR}=1.059,95 \% \mathrm{CI}, 1.025-1.093, P<$ $0.001)$ and history of coronary artery disease $(\mathrm{OR}=2.003$, $955 \mathrm{CI}, 1.16-3.457, P=0.013)$ were associated with rtPA administration while increasing age $(\mathrm{OR}=0.979,95 \% \mathrm{CI}$, 0.961-0.996, $P=0.017)$, higher INR (OR $=0.146,95 \% \mathrm{CI}$, $0.032-0.665, P=0.013)$, history of previous stroke ( $\mathrm{OR}=$ $0.39,95 \% \mathrm{CI}, 0.223-0.68, P=0.001$ ), and renal insufficiency $(\mathrm{OR}=0.153,95 \% \mathrm{CI}, 0.046-0.508, P=0.002)$ are associated with rtPA exclusion. The ROC curve reveals a strong prediction of the logistic regression model (Fig. 3), AUROC = 0.650 (95\% CI, 0.602-0.699, $P<0.005$ ). An adjusted analysis for the telestroke subgroup (Table 6), reveals that only direct admission is associated with rtPA administration, (OR $=4.083,95 \% \mathrm{CI}, 1.322-12.611, P=0.014)$, and the predictive model power of the logistic regression was strong (Fig. 4), AUROC $=0.678$ (95\% CI, 0.639-0.718, $P<0.05$ ).

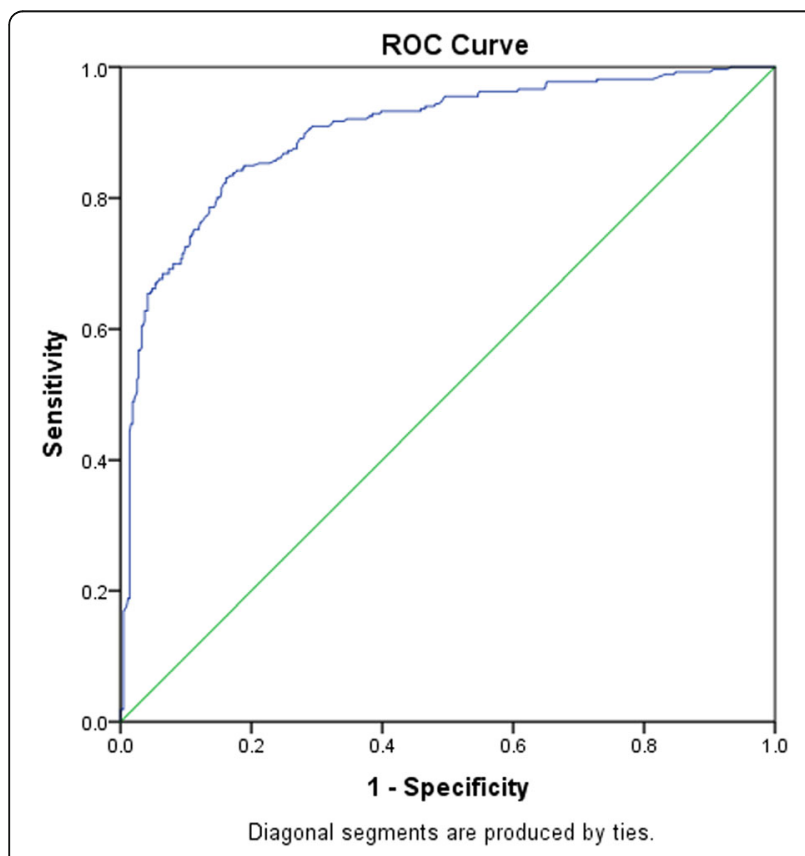

Fig. 1 ROC curve to analyze the predictive power of the logistic regression presented in Table 2 . AUROC $=0.900(0.875-0.924, P<0.05)$

\section{Discussion}

In a population of hypertensive stroke patients, we found patients that present with obesity, who are directly admitted to the hospital, and received rtPA have higher odds of being associated with the telestroke, while those with a higher systolic blood pressure were associated with the non telestroke. After adjusting for comorbidities without sorting by telestroke and non telestroke, we found that irrespective of whether patients were treated in the telestroke or non telestroke, the telestroke represents the strongest predictor of rtPA administration followed by a direct admission and NIH stroke scale scores. However, a higher INR, history of previous stroke and renal insufficiency were predictors of exclusion of hypertensive stroke patients from rtPA.

In the univariate analysis for the non telestroke, a higher NIH stroke scale score and history of coronary artery disease were associated with inclusion for rtPA while increasing age, higher INR, history of previous stroke, and renal insufficiency were all associated with rtPA exclusion. Following an adjustment for the baseline clinical variables in the telestroke subgroup, direct admission was the only factor associated with rtPA administration with a strong predictive power in the regression

Table 4 A stepwise regression model to elucidate clinical factors more associated rtPA inclusion in the total study population

\begin{tabular}{lllll}
\hline & B Value & Adj. Odds Ratio & Wald & $P$ Value \\
\hline NIH Stroke Scale Score & 0.045 & $1.046(1.016-1.076)$ & 9.320 & $0.002^{*}$ \\
INR & -1.595 & $0.203(0.06-0.691)$ & 6.502 & $0.011^{*}$ \\
Previous Stroke & -0.742 & $0.476(0.293-0.775)$ & 8.934 & $0.003^{*}$ \\
Renal Insufficiency & -1.048 & $0.351(0.143-0.858)$ & 5.271 & $0.022^{*}$ \\
Direct Admission & 1.517 & $4.557(1.772-11.721)$ & 9.900 & $0.002^{*}$ \\
Telestroke & 1.650 & $5.204(2.582-10.492)$ & 21.262 & $<0.001^{*}$ \\
Constant & 1.490 & 4.439 & 4.731 & $0.03^{*}$
\end{tabular}

Positive $B$ values ( $A d j, O R>1$ ) denote variables more associated with rtPA inclusion while negative $B$ values (Adj. $O R<1$ ) denote variables more associated with rtPA exclusion. Multicollinearity and interactions among independent variables were checked. Hosmer-Lemeshow test $(P=0.006)$, Cox \& Snell $\left(R^{2}=0.260\right)$, classification table (overall correctly classified percentage $=75.3 \%$ ) were applied to check the model fitness ${ }^{*} P<0.05$ 


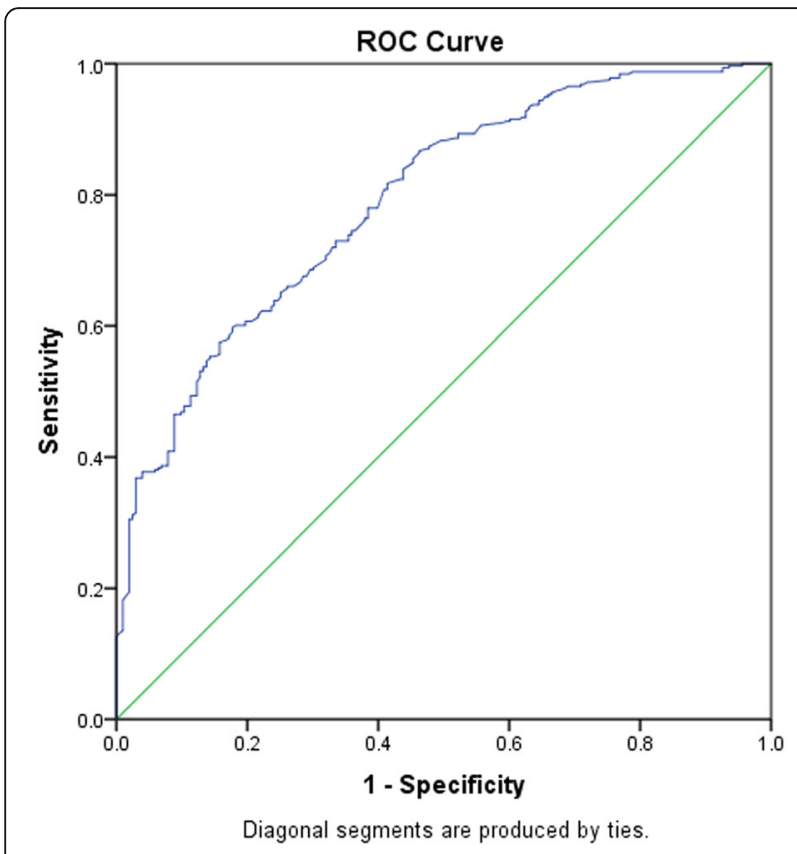

Fig. 2 ROC curve to analyze the predictive power of the logistic regression presented in Table 4. AUROC $=0.792(0.753-0.830, P<0.05)$

model. Clearly, the higher proportion of clinical risk factors associated with exclusion in the non telestroke when compared with the telestroke is not unconnected with the exclusion of more hypertensive stroke patients from rtPA; because though the non telestroke admitted more patients (62\% vs $38 \%$ ) when compared with the telestroke, more patients (61.3\% vs $38.7 \%)$ were excluded from rtPA. We observed that the telestroke admitted fewer patients, excluded $11 \%$ and administered thrombolysis therapy to $89 \%$ of hypertensive stroke patients. It is possible that the telestroke technology provides a real-world clinical experience with less stringent or restrictive exclusion criteria focused more on

Table 5 A stepwise regression model to elucidate clinical factors more associated rtPA inclusion in the non-telestroke population

\begin{tabular}{lllll}
\hline & B Value & Adj. Odds Ratio & Wald & $P$ Value \\
\hline Increasing Age & -0.022 & $0.979(0.961-0.996)$ & 5.726 & $0.017^{*}$ \\
NIH Stroke Scale Score & 0.057 & $1.059(1.025-1.093)$ & 12.190 & $<0.001^{*}$ \\
INR & -1.927 & $0.146(0.032-0.665)$ & 6.180 & $0.013^{*}$ \\
Coronary Artery Disease & 0.695 & $2.003(1.16-3.457)$ & 6.222 & $0.013^{*}$ \\
Previous Stroke & -0.942 & $0.39(0.223-0.68)$ & 10.984 & $0.001^{*}$ \\
Renal Insufficiency & -1.879 & $0.153(0.046-0.508)$ & 9.387 & $0.002^{*}$ \\
Constant & 3.122 & 22.700 & 9.628 & $0.002^{*}$
\end{tabular}

Positive $B$ values ( $\mathrm{Adj}, \mathrm{OR}>1$ ) denote variables more associated with rtPA inclusion while negative $B$ values (Adj. OR $<1$ ) denote variables more associated with rtPA exclusion. Multicollinearity and interactions among independent variables were checked. Hosmer-Lemeshow test $(P=0.854)$, Cox \& Snell $\left(R^{2}=0.159\right)$, classification table (overall correctly classified percentage $=67 \%$ ) were applied to check the model fitness

${ }^{*} P<0.05$

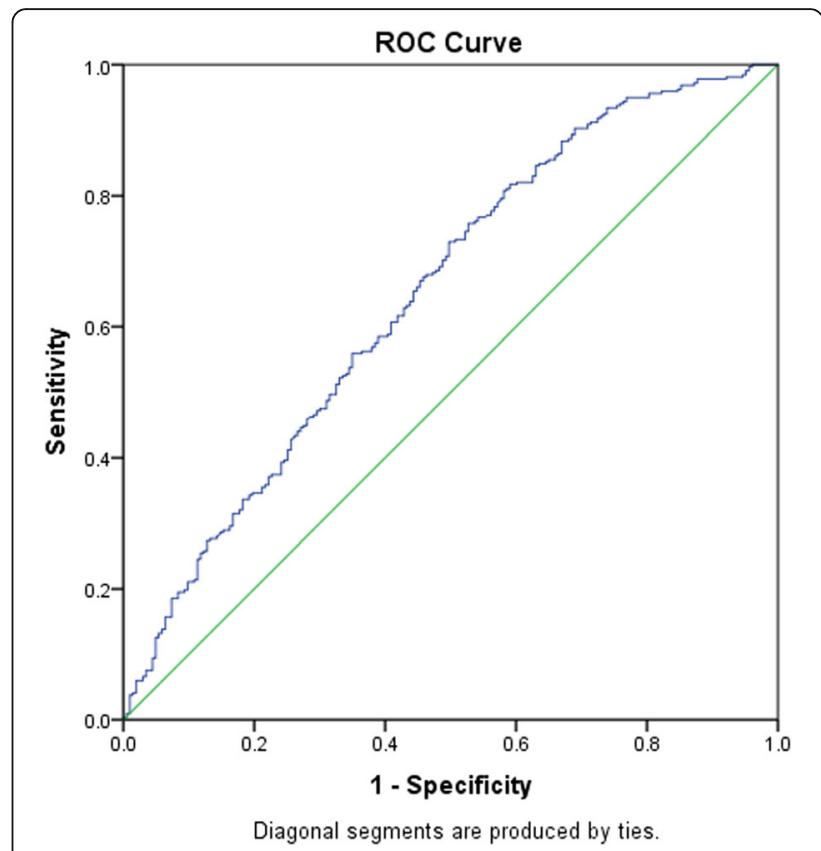

Fig. 3 ROC curve to analyze the predictive power of the logistic regression presented in Table 5. AUROC $=0.650(0.602-0.699, P<0.05)$

benefit-to-risk ratio for thrombolysis therapy. The fact that the non telestroke group had more clinical risk factors associated with exclusion from thrombolysis indicates that stricter criteria were used when compared with the telestroke group, which had less stringent criteria on an individual basis. In a whole stroke hypertensive population telestroke was the strongest predictor of rtPA administration, followed by a direct admission and higher NIH stroke scale score respectively. Our model predicted a direct association of telestroke with the highest odds for the inclusion of hypertensive stroke patients for thrombolysis therapy. Other studies have indirectly linked the telestroke technology with the improvement of stroke care in rural health care centers [13], increased rate of rtPA administration [38, 43] and improvement of the timeliness of rtPA administration [39, 40]. Our finding of a strong association of telestroke with thrombolysis may reflect the fast response for the early recommendation of administration of rtPA in the hub station and faster administration in the spoke station resulting in significant increases in the rates of rtPA utilization at the spoke hospitals. There is a growing

Table 6 A stepwise regression model to elucidate clinical factors more associated rtPA inclusion in the telestroke population

\begin{tabular}{lllll}
\hline & B Value & Adj. Odds Ratio & Wald & $P$ Value \\
\hline Direct Admission & 1.407 & $4.083(1.322-12.611)$ & 5.979 & $0.014^{*}$ \\
Constant & 1.569 & 4.800 & 20.363 & $<0.001^{*}$ \\
\hline
\end{tabular}

${ }^{*} P<0.05$ 


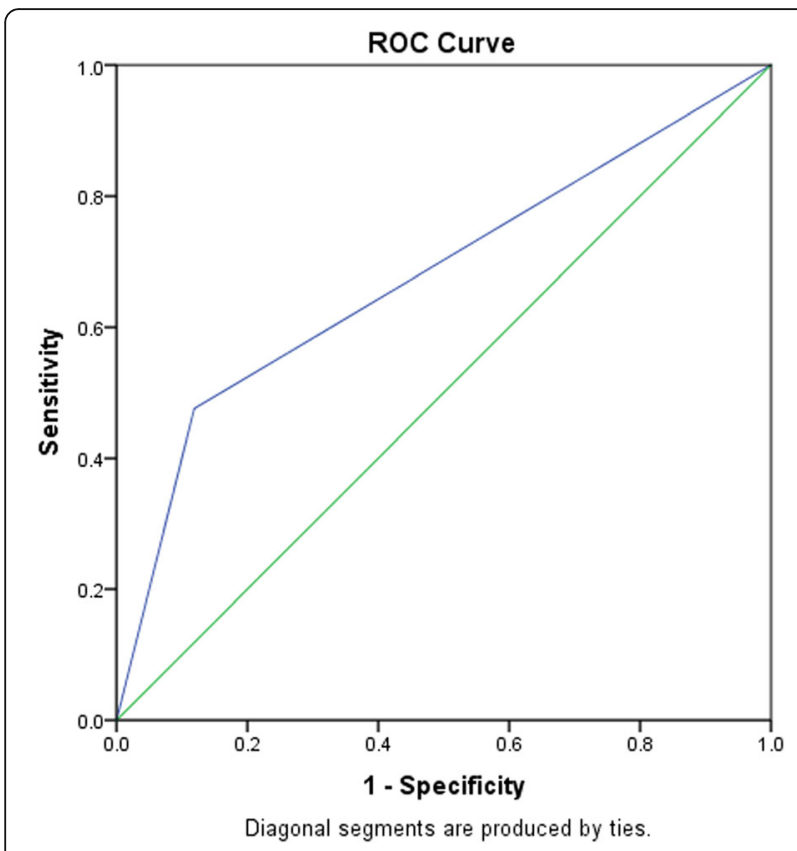

Fig. 4 ROC curve to analyze the predictive power of the logistic regression presented in Table 6. AUROC $=0.678(0.639-0.718, P<0.05)$

evidence that telemedicine services offer hypertensive patients the access to diagnostic measures $[41,44]$ that might not be available with usual bedside evaluation care. This indicates that telemedicine has the ability to deconstruct the typical traditional model in the standard clinical consultation [29]. This might impact the management of hypertension in ischemic stroke beyond the use of antiHTN in the telestroke with some form of additional support compared with usual care not common in the non telestroke. In this context, the telestroke could be a part of the solution to improve the rate of thrombolysis in hypertensive stroke patients.

In the univariate analysis, the effect of direct admission was not significant in both the telestroke and non telestroke settings. Following an adjusted analysis for both the telestroke and non telestroke, a direct admission of patients was associated with thrombolysis in the telestroke setting. The significant association of direct admission with thrombolysis was sustained in the whole stroke population following adjustment for baseline demographic and clinical risk factors. In general, direct admission is known to be associated with shorter onset-to-needle time and better outcome in patients with acute ischemic stroke undergoing thrombolysis [45-50]. This is because a direct admission to the telestroke could reduce the door-to-needle time for thrombolysis to within an hour in more than $70 \%$ of acute ischemic stroke patients and less than $50 \mathrm{~min}$ in more than $45 \%$ of stroke patients [51-53]. Many methods have been proposed to reduce door-to-needle time for thrombolysis, including in-hospital system-level centralized telestroke care $[10,13,16,54-58]$. Therefore, it is possible that in hypertensive stroke patients, telestroke may be associated with improved thrombolysis therapy use by facilitating a direct admission that improves the time to receive thrombolysis therapy. Such a time improvement could be due to the reduction of the time between hub stroke neurologist's and spoke clinician's involvement in the management, treatment, and implementation of the protocol to mix rtPA and administer thrombolysis to hypertensive stroke patients.

Our finding that INR, previous stroke and increased age (> 80 years) are associated with exclusion of hypertensive stroke patients from rtPA have been reported as exclusion variables in acute ischemic stroke patients [3, 59-66]. In general, existing guidelines regarding INR results are intended to prevent hemorrhages and, specifically intracranial hemorrhage which is the most dreaded complication of rtPA in patients taking oral anticoagulants. In particular, the AHA/ASO guidelines [67] recommend that, the administration of rtPA can be initiated before results of coagulation tests or platelet counts are available, unless a bleeding is observed after the patient had already received heparin or other anticoagulants. However, if rtPA protocol has been initiated and the results of the coagulation tests or platelet counts indicate that INR $>1.7$ or a PT $>15 \mathrm{~s}$ by local protocol of rtPA administration, then the procedure should be stopped [68]. This might have contributed to the exclusion of more patients in the non telestroke in our current study.

We found that hypertensive stroke patients with age > 80 years are more likely to be excluded more from rtPA in the non telestroke setting. Observational studies aimed at monitoring the safety of rtPA provided comparative results on stroke outcome between patients $>80$ years and those $<80$ years $[63,69-72]$. Findings reveal that $>80$ years are less likely to achieve good outcomes compared with $<80$ years. Most of the observational studies evaluated age differences between older and younger patients receiving rtPA. In our adjusted analysis, more hypertensive stroke patients $>80$ years are more likely to be excluded from rtPA compared with $>80$ years that received rtPA in the non telestroke. In general, patients with an elevated INR and a previous stroke within the last 3 months are associated with contraindications for rtPA in other studies $[38,64,73-77]$. Therefore, the combined effect of old age coupled with elevated INR and a previous stroke within the last 3 months played a role in the exclusion criteria in acute ischemic stroke patients with incidence hypertension in the current study.

There are limitations in this study. This is a single healthcare system initiative with retrospective data collection. Therefore, there is the tendency for selection bias because of the lack of an experimental design that 
allows for randomization, especially at telestroke or non telestroke settings. For this reason, our finding cannot be generalized to other hospital settings. Since our data is pretreatment, and post treatment only until discharge from the hospital, it did not include longitudinal data for a follow-up to determine the effect of direct admission on post rtPA outcome. Moreover, information about the management of hypertension and the time for direct and indirect admission was not included in our analysis, preventing comparison of onset-to-door time between indirect and direct admission. The number ischemic stroke patients that needed antihypertensive treatment before rtPA were not included. However, our model associated telestroke with thrombolysis in providing clinical practice with less restrictive exclusions focused more on benefit-to-risk ratio for thrombolysis therapy in stroke patients with hypertension.

\section{Conclusion}

In summary, telestroke was the strongest predictor of rtPA administration, followed by a direct admission and higher NIH stroke scale score. More studies are necessary to determine how identified exclusion risk factors in the non telestroke setting can be improved, including a direct transfer of hypertensive stroke patients to health care centers with a direct access to thrombolysis.

\section{Abbreviations \\ AHA: American Heart Association; AUROC: Area under the curve; CAD: Coronary artery disease; CHF: Congestive heart failure; Cl: Confidence interval; GHS: Greenville Health System; GWTG: Get with the guideline; MAP: Mean arterial pressure; NIH scores: National Institute of Health scores; OR: Adjusted odd ratio; PVD: Peripheral vascular disease; ROC: Receiver operator characteristic; rtPA: Recombinant tissue plasminogen activator; TIA: Trans ischemic attack}

\section{Acknowledgements}

We thank the stroke unit of Greenville Health system for helping in the data collection.

\section{Funding}

This study was funded by the Fullerton Foundation. The funders had no role in study design, data collection and analysis, decision to publish or preparation of the manuscript.

\section{Availability of data and materials}

All materials are available for use from the corresponding author.

\section{Authors' contributions}

$L B, J D, A P$ and TIN designed the concept, experimental design and data analysis and critically revised the drafts read and approved the last version of this manuscript.

\section{Ethics approval and consent to participate}

The study did not involve patients and no consent was required. This study was approved by the Institutional Review Board of Greenville Health System and the institutional Committee for Ethics.

\section{Consent for publication}

Not applicable.

\section{Competing interests}

Dr. Thomas Nathaniel is a member of the editorial board (Associate Editor).

\section{Publisher's Note}

Springer Nature remains neutral with regard to jurisdictional claims in published maps and institutional affiliations.

Received: 24 July 2018 Accepted: 26 November 2018

Published online: 21 December 2018

\section{References}

1. Rubin MN, Wellik KE, Channer DD, Demaerschalk BM. Systematic review of Telestroke for post-stroke care and rehabilitation. Curr Atheroscleros Rep. 2013:15(8):343.

2. Rubin MN, Demaerschalk BM. The use of telemedicine in the management of acute stroke. Neurosurg Focus. 2014;36(1):E4.

3. Wormack L, Ubah C, Orfanakos B, Nathaniel T. Acute ischemic stroke: Can clinical risk factors exclude more women than men from thrombolysis therapy. Atlas of Science. 2018;5(23);34.

4. Demaerschalk BM, Switzer JA, Xie JP, Fan LY, Villa KF, Wu EQ. Cost utility of hub-and-spoke Telestroke networks from societal perspective. Am J Manag Care. 2013;19(12):976-85.

5. Switzer JA, Demaerschalk BM, Xie JP, Fan LY, Villa KF, Wu EQ. Costeffectiveness of hub-and-spoke Telestroke networks for the Management of Acute Ischemic Stroke from the Hospitals' perspectives. Circ Cardiovasc Qual Outcomes. 2013;6(1):18-26.

6. Cadilhac DA, Moloczii N, Denisenko S, Dewey H, Disler P, Winzar B, Mosley I, Donnan GA, Bladin C. Establishment of an effective acute stroke telemedicine program for Australia: protocol for the Victorian stroke telemedicine project. Int J Stroke. 2014;9(2):252-8.

7. Levine SR, Gorman M. "TeleStroke" - the application of telemedicine for stroke. Stroke. 1999;30(2):464-9.

8. Meretoja A, Strbian D, Mustanoja S, Tatlisumak T, Lindsberg PJ, Kaste M. Reducing in-hospital delay to 20 minutes in stroke thrombolysis. Neurology. 2012;79(4):306-13

9. Bagot KL, Bladin CF, Vu M, Kim J, Hand PJ, Campbell B, Walker A, Donnan GA, Dewey HM, Cadilhac DA, et al. Exploring the benefits of a stroke telemedicine programme: an organisational and societal perspective. J Telemed Telecare. 2016;22(8):489-94.

10. Bhatt A, Lesko A, Lucas L, Kansara A, Baraban E. Patients with low National Institutes of Health stroke scale scores have longer door-to-needle times: analysis of a Telestroke network. J Stroke Cerebrovasc Dis. 2016;25(9):2253-8.

11. Blacquiere D, Lindsay MP, Foley N, Taralson C, Alcock S, Balg C, Bhogal S, Cole J, Eustace M, Gallagher P, et al. Canadian stroke best practice recommendations: telestroke best practice guidelines update 2017. Int J Stroke. 2017;12(8):886-95.

12. Dumitrascu OM, Demaerschalk BM. Telestroke. Curr Cardiol Rep. 2017;19(9):85.

13. Nguyen-Huynh MN, Klingman JG, Avins AL, Rao VA, Eaton A, Bhopale S, Kim AC, Morehouse JW, Flint AC, Team KSF. Novel telestroke program improves thrombolysis for acute stroke across 21 hospitals of an integrated healthcare system. Stroke. 2018:49(1):133-9.

14. Rodriguez-Castro E, Jose Vazquez-Lima M, Rodriguez-Yanez M, Verde L, Castillo J. Stroke care in Galicia: telemedicine in the early, multidisciplinary treatment of all acute stroke cases. Emergencias. 2018;30(1):54-61.

15. Sharma R, Fleischut $P$, Barchi D. Telemedicine and its transformation of emergency care: a case study of one of the largest US integrated healthcare delivery systems. Int J Emerg Med. 2017;10:21.

16. Sanders KA, Patel R, Kiely JM, Gwynn MW, Johnston LH. Improving telestroke treatment times in an expanding network of hospitals. J Stroke Cerebrovasc Dis. 2016;25(2):288-91.

17. Wapshott T, Blum B, Williams K, Nathaniel TI. Investigation of gender differences and exclusive criteria in a diabetic acute ischemic stroke population treated with recombinant tissue-type plasminogen activator (rtPA). J Vasc Interv Neurol. 2017;9(6):26-32.

18. Nathaniel IT, Williams J, Fazzone F, Yi S, Morris G, Black L, Fredwall M, Staford C, Adkins A, Polk S. Contraindications and exclusion criteria in guidelines for Rt-pa in acute ischemic stroke: can the new Aha/asa guideline expand the use of Rt-pa? In: Hypertension; 2016. p. 245.

19. Polk S, Stafford C, Adkins A, Efird J, Colello M, Nathaniel IT. Contraindications with recombinant tissue plasminogen activator (rt-PA) in acute ischemic stroke population. Neurol Psychiatry Brain Res. 2018;27:6-11.

20. Thomas IN, Gainey J, Wormack J, Brechtel L. Functional outcome model for a Telestroke-guided tissue plasminogen activator treatment of stroke. Int Stroke Conf Proc. 2018;1:45. 
21. Gainey J, Wormack L, Brechtel L, Nathaniel IT. A functional outcome mode for a telestroke-guided tissue plasminogen activator treatment of stroke patients. Stroke. 2018;49(Suppl 1):P89.

22. Gainey J, Brecthtel J, Blum B, Keels A, Madeline L, Lowther E, Nathaniel IT. Functional outcome measures of recombinant tissue plasminogen activator-treated stroke patients in the telestroke technology. J Exp Neurosci. 2018;12:1-11.

23. Gainey J, Brechtel L, Konklin S, Madeline L, Lowther E, Blum B, Nathaniel TI. In a stroke cohort with incident hypertension; are more women than men likely to be excluded from recombinant tissue-type plasminogen activator (rtPA)? J Neurol Sci. 2018;387:139-46.

24. Gainey J, Blum B, Bowie B, Cooley K, Madeline L, Ervin EL, Nathaniel TI. Stroke and dyslipidemia: clinical risk factors in the telestroke versus nontelestroke. Lipids Health Dis. 2018;17(1):226.

25. Cochran T, Chaves J, Fulmer E, Blackhurst D, Sternberg S, Leacock R, Nathaniel T. Analysis of comorbidities in ischemic stroke patients. Neurology. 2014;82(10 Supplement):278.

26. Gasecki D, Coca A, Cunha P, Hering D, Manios E, Lovic D, Zaninelli A, Sierra C, Kwarciany M, Narkiewicz K, et al. Blood pressure in acute ischemic stroke: challenges in trial interpretation and clinical management: position of the ESH working group on hypertension and the brain. J Hypertens. 2018;36(6): $1212-21$.

27. Jamali AA, Jamali GM, Jamali AA, Khaskhali KB, Jamali NH, Rajput AA Tanwani BM, Jamali NM. Association of hemorrhagic stroke patients with hypertension, diabetes mellitus and smoking in Nawabshah, Sindh, Pakistan. Indo Am J Pharm Sci. 2018;5(5):3720-5.

28. Plante TB, Long DL, Howard G, Carson AP, Howard VJ, Judd SE, Jenny NS, Zakai NA, Cushman M. Contribution of C-reactive protein to racial disparties in incident hypertension: the REasons for geographic and racial differences in stroke (REGARDS). Circulation. 2018;137:AP181.

29. Gainey J, Blum B, Bowie B, Cooley K, Madeline L, Lowther E, Nathaniel IT. Stroke with dyslipidemia: clinical risk factors in the telestroke versus nontelestroke. Lipids Health Dis. 2018;4(87):123-48.

30. Bladin CF, Cadilhac DA. Effect of Telestroke on emergent stroke care and stroke outcomes. Stroke. 2014;45(6):1876-80.

31. Nathaniel IT, Gainey J, Blum B, Montgomery C. Clinical risk factors in thrombolysis therapy: telestroke versus nontelestroke. J Stroke Cerebrovasc Dis. 2018;27(9):2524-33.

32. Lisk D, Grotta J, Lamki L, Tran H, Taylor J, Molony D, Barron B. Should hypertension be treated after acute stroke - a randomized controlled trial using single-photon emission computed-tomography. Ann Neurol. 1993; 34(2):253-4.

33. Novak V, Hu K, Desrochers L, Novak P, Caplan L, Lipsitz L, Selim M. Cerebral flow velocities during daily activities depend on blood pressure in patients with chronic ischemic infarctions. Stroke. 2010;41(1):61-6.

34. Cheng A, Brown MM, Richards T. Prevalence of carotid artery stenosis in patients with transient ischaemic attack or ischaemic stroke: a large prospective case series, systematic review and metaregression analysis. Eur J Neurol. 2017:24:36.

35. Gacon J, Badacz R, Stepien E, Karch I, Enguita FJ, Zmudka K, Przewlocki T, Kablak-Ziembicka A. Diagnostic and prognostic microRNAs in ischaemic stroke due to carotid artery stenosis and in acute coronary syndrome: a four-year prospective study. Kardiol Pol. 2018; 76(2):362-9.

36. Lattanzi S, Silvestrini M. Carotid artery stenosis and PERI-operative stroke in cardiac surgery. A bridge between heart and brain. J Neurol Sci. 2017;382:168-9.

37. Fleming T, Averkamp B, Sulivan J, Nathaniel T. Effect of Antihypertensive Medications on rt-PA in Stroke Patients; Improved or Poor Outcomes? Interv Neurol. 2017:6(supple 1):70.

38. Fazzone B, Morris G, Black LA, Williams JA, Leacock R, Sternberg S, Blackhurst D, Nelson A, Nathaniel IT. Exclusion and inclusion criteria for thrombolytic therapy in an ischemic stroke population.e 4(2): 1112. J Neurol Disord Strok. 2016;4(2):1-5.

39. Lee VH, Cutting S, Song SY, Cherian L, Diebolt E, Bock J, Conners JJ. Participation in a tele-stroke program improves timeliness of intravenous thrombolysis delivery. Telemed E-Health. 2017;23(1):63-5.

40. Lawson T, Brown I, Westerkam D, Blackhurst D, Sternberg S, Leacock R, Nathaniel IT: A new diagnostic tool for measuring the effectiveness of Intravenous TIssue Plasminogen Activator ( $r-\mathrm{pa}$ ) In The Treatment Of Acute Ischemic Stroke International stroke conference 2015, 4(6):346.
41. Reynolds M, Blum B, Brechtel L, Gainey G, Nathaniel IT. Clinical risk factors associated with functional outcomes of thrombolytic therapy in stroke and non-stroke units. J Exp StrokeTransl Med. 2017;1:22-8.

42. Jackson JH, Sobolski J, Krienke R, Wong KS, Frech-Tamas F, Nightengale B. Blood pressure control and pharmacotherapy patterns in the United States before and after the release of the joint National Committee on the prevention, detection, evaluation, and treatment of high blood pressure (JNC 7) guidelines. J Am Board Fam Med. 2008;21(6):512-21.

43. Bozorgui S, Wu TC, Bambhroliya AB, Malazarte RM, Ankrom CM, Cossey TD, Trevino AD, Savitz SI, Jagolino-Cole AL. Similar outcomes for patients treated with off-label vs on-label tissue plasminogen activator via telestroke. Stroke. 2018;49 (Suppl 1):AWP225-AWP225.

44. Omboni S, Ferrari R. The role of telemedicine in hypertension management: focus on blood pressure Telemonitoring. Curr Hypertens Rep. 2015;17(4):535.

45. Chalouhi N, Dressler JA, Kunkel ESI, Dalyai R, Jabbour P, Gonzalez LF, Starke RM, Dumont AS, Rosenwasser R, Tjoumakaris S. Intravenous tissue plasminogen activator Administration in Community Hospitals Facilitated by Telestroke service. Neurosurgery. 2013;73(4):667-71.

46. Kim DH, Bae HJ, Han MK, Kim BJ, Park SS, Park TH, Lee KB, Kang K, Park JM, Ko $Y$, et al. Direct admission to stroke centers reduces treatment delay and improves clinical outcome after intravenous thrombolysis. J Clin Neurosci. 2016;27:74-9.

47. Kim DH, Nah HW, Park HS, Choi JH, Kang MJ, Huh JT, Cha JK. Impact of prehospital intervention on delay time to thrombolytic therapy in a stroke center with a systemized stroke code program. J Stroke Cerebrovasc Dis. 2016;25(7):1665-70.

48. Sauser K, Levine DA, Nickles AV, Reeves MJ. Hospital variation in thrombolysis times among patients with acute ischemic stroke the contributions of door-to-imaging time and imaging-to-needle time. JAMA Neurol. 2014;71(9):1155-61.

49. Xian Y, Xu HL, Lytle B, Blevins J, Peterson ED, Hernandez AF, Smith EE, Saver $J$, Messe SR, Paulsen M, et al. Use of strategies to improve door-to-needle times with tissue-type plasminogen activator in acute ischemic stroke in clinical practice: findings from target: stroke. Circ Cardiovasc Qual Outcomes. 2017;10(1). https://doi.org/10.1161/CIRCOUTCOMES.116.003227.

50. Scalise M, Bailes B, Brechtel L, Conn Z, Gainey BS, Nathaniel T. A Predictive Model to Measure Treatment Outcomes in a Stroke Belt State. Interv Neurol. 6( suppl 1):90.

51. Bajkiewicz CT, Kalafut M, Olson L, Afshar K. 'Expedite for Thrombolytic' patient designation and early ordering for improved door-to-needle times in acute ischemic stroke. Stroke. 2015:46:ATP346.

52. Fonarow GC, Cox M, Smith EE, Saver JL, Reeves MJ, Bhatt DL, Suter R, Schulte PJ, Xian Y, Hernandez AF, et al. Improving door-to-needle times in acute ischemic stroke: interim findings from target: stroke phase II. Stroke. 2016:47:A115

53. Gurav SK, Zirpe KG, Wadia RS, Naniwadekar A, Pote PU, Tungenwar A, Deshmukh AM, Mohopatra S, Nimavat B, Surywanshi P. Impact of "stroke code"-rapid response Team: an attempt to improve intravenous thrombolysis rate and to shorten door-to-needle time in acute ischemic stroke. Indian J Crit Care Med. 2018;22(4):243-8.

54. Bladin CF, Molocijz N, Ermel S, Bagot KL, Kilkenny M, Vu M, Cadilhac DA, Investigators VSTP. Victorian stroke telemedicine project: implementation of a new model of translational stroke care for Australia. Intern Med J. 2015; 45(9):951-6

55. Martinez-Sanchez P, Miralles A, de Barros RS, Prefasi D, Sanz-Cuesta BE, Fuentes B, Ruiz-Ares G, Martinez-Martinez M, Minano E, Arevalo-Manso JJ, et al. The effect of telestroke systems among neighboring hospitals: more and better? The Madrid Telestroke project. J Neurol. 2014;261(9):1768-73.

56. Moreno A, Schwamm LH, Siddiqui KA, Viswanathan A, Whitney C, Rost N, Zachrison KS. Frequent hub-spoke contact is associated with improved spoke hospital performance: results from the Massachusetts General Hospital Telestroke network. Telemed E-Health. 2018;24(9):678-83.

57. Nardetto L, Dario C, Tonello S, Brunelli MC, Lisiero M, Carraro MG, Saccavini C, Scannapieco G, Giometto B. A one-to-one telestroke network: the first Italian study of a web-based telemedicine system for thrombolysis delivery and patient monitoring. Neurol Sci. 2016:37(5):725-30.

58. Ranta A, Lanford J, Busch S, Providence C, Iniesta I, Rosemergy I, Wilson A, Cariga P, Richmond V, Gommans J. Impact and implementation of a sustainable regional telestroke network. Intern Med J. 2017;47(11):1270-5.

59. Reiff T, Michel P. Reasons and evolution of non-thrombolysis in acute ischaemic stroke. Emerg Med J. 2017;34(4):219-26. 
60. Isobel JH, Wass S, Pepper E. Stroke in older survivors of ischemic stroke: standard care or something different. Geriatrics. 2017;2(18):1-13.

61. Wapshott T, Blum B, Williams K, Nathaniel IT. Investigation of gender differences and exclusive criteria in a diabetic acute ischemic stroke population treated with recombinant tissue-type plasminogen activator (rtPA). J Vasc Interv Neurol. 2017;9(3):26-32.

62. Fredwall M, Sternberg S, Blackhurst D, Lee A, Leacock R, Nathaniel TI. Gender differences in exclusion criteria for recombinant tissue-type plasminogen activator. J Stroke Cerebrovasc Dis. 2016;25(11):2569-74.

63. Lawson TR, Brown IE, Westerkam DL, Blackhurst DW, Sternberg S, Leacock R, Nathaniel TI. Tissue plasminogen activator (rt-PA) in acute ischemic stroke: outcomes associated with ambulation. Restor Neurol Neurosci. 2015;33(3): $301-8$.

64. Colello MJ, Ivey LE, Gainey J, Faulkner RV, Johnson A, Brechtel L, Madeline L, Nathaniel TI. Pharmacological thrombolysis for acute ischemic stroke treatment: gender differences in clinical risk factors. Adv Med Sci. 2018;63(1): $100-6$.

65. Nathaniel TI, Cochran T, Chaves J, Fulmer E, Sosa C, Yi S, Fredwall M, Sternberg S, Blackhurst D, Nelson A, et al. Co-morbid conditions in use of recombinant tissue plasminogen activator (rt-PA) for the treatment of acute ischaemic stroke. Brain Inj. 2016;30(10):1261-5.

66. Nathaniel IT, Wormack L, Gainey J. Development of a new predictive model for thrombolysis in the telestroke for hypertensive acute ischemic stroke patients. In: 11th world stroke congress: 2018; 2018.

67. Jauch EC, Saver JL, Adams HP, Bruno A, Connors JJ, Demaerschalk BM, Khatri P, McMullan PW, Qureshi Al, Rosenfield K, et al. Guidelines for the early Management of Patients with Acute Ischemic Stroke a Guideline for healthcare professionals from the American Heart Association/American Stroke Association. Stroke. 2013;44(3):870-947.

68. Bruch TP, Mendes DC, Pedrozo JC, Figueiredo L, Novak EM, Zetola VF, Lange MC. Is point-of-care accurate for indicating thrombolysis in anticoagulated patients on oral anticoagulation treatments? Arq Neuropsiquiatr. 2014;72(7): 487-9.

69. Davis SM, Donnan GA, Parsons MW, Levi C, Butcher KS, Peeters A, Barber PA, Bladin C, De Silva DA, Byrnes $\mathrm{G}$, et al. Effects of alteplase beyond $3 \mathrm{~h}$ after stroke in the Echoplanar imaging thrombolytic evaluation trial (EPITHET): a placebo-controlled randomised trial. Lancet Neurol. 2008;7(4):299-309.

70. Brown MD. Clinical policy: use of Intravenous Tissue Plasminogen Activator for the Management of Acute Ischemic Stroke in the Emergency Department (vol 66, pg 322, 2015). Ann Emerg Med. 2017;70(5):758.

71. Grp ISTC. The benefits and harms of intravenous thrombolysis with recombinant tissue plasminogen activator within $6 \mathrm{~h}$ of acute ischaemic stroke (the third international stroke trial IST-3): a randomised controlled trial (vol 379, pg 2352, 2012). Lancet. 2012;380(9843):730.

72. Okon M, Adebobola NI, Julius S, Adebimpe O, Taiwo AO, Akinyemi A, Thomas NI. Stroke incidence and case fatality rate in an urban population. J Stroke Cerebrovasc Dis. 2015;24(4):771-7.

73. Demaerschalk. Scientific rationale for the inclusion and exclusion criteria for intravenous Alteplase in acute ischemic stroke: a statement for healthcare professionals from the American Heart Association/American Stroke Association (vol 47, pg 581, 2016). Stroke. 2016;47(11):E262.

74. Buchan AM. Why are stroke patients excluded from tPA therapy? An analysis of patient eligibility - reply. Neurology. 2001;57(9):1739-40.

75. Chuanjie W, Di W, Jian C, Chuanhui L, Xunming J. Why not intravenous thrombolysis in patients with recurrent stroke within 3 months? Aging Dis. 2018;9(2):309-16

76. Polk SR, Stafford C, Adkins A, Efird J, Colello M, Nathaniel IT. Contraindications with recombinant tissue plasminogen activator (rt-PA) in acute ischemic stroke population. Neurol Psychiatry Brain Res. 2017;29.

77. Cochran T, Chaves J, Fulmer E, Blackhurst D, Sternberg S, Leacock R, Nathaniel T. Analysis of comorbidities in ischemic stroke patient. Neurology. 2014;82(10):P6.278

Ready to submit your research? Choose BMC and benefit from:

- fast, convenient online submission

- thorough peer review by experienced researchers in your field

- rapid publication on acceptance

- support for research data, including large and complex data types

- gold Open Access which fosters wider collaboration and increased citations

- maximum visibility for your research: over $100 \mathrm{M}$ website views per year

At BMC, research is always in progress.

Learn more biomedcentral.com/submissions 